Pecvnia, 10 (2010), pp. 265-297

\title{
Las relaciones causales recíprocas del binomio Partida Doble-desarrollo de Occidente: La Teneduría de Libros de los mercaderes medievales
}

Recibido: Noviembre 2009 Aceptado: Diciembre 2009
J. Lanero Fernández

juan.lanero@unileon.es Universidad de León

Fac. de Ciencias Económicas y Empresariales Campus de Vegazana, $\mathrm{s} / \mathrm{n}$ 24071 León (España)
Mucho se ha escrito sobre la aportación de la Partida Doble al desarrollo económico de Occidente. El presente trabajo pretende presentar un análisis detallado del papel que desempeñó la contabilidad en la génesis del capitalismo. La contabilidad, como herramienta de gestión, fue un factor clave en la organización comercial.

Después de ofrecer una visión panorámica de la organización del comercio en la época medieval y de considerar varias acepciones del
Thousands of pages have been written about the double-entry contribution to the economic development of the Western world. The present paper tries to present a detailed analysis on the role played by Accounting in the genesis of capitalism. Accounting, as a management tool, was a key factor in the commercial organization.

After having offered a general view about the organization of commerce in the Medieval

Conferencia pronunciada en las XV Jornadas de Epistemología de las Ciencias Económicas, Universidad de Buenos Aires (Argentina) en octubre de 2009 bajo el título "Mercaderes sedentarios, poderdantes y factores: Reflexiones sobre la contribución de la Partida Doble al desarrollo económico de Occidente". 
concepto de partida doble, el estudio concluye que ésta fue al mismo tiempo causa y efecto de la transición de los mercaderes itinerantes a sedentarios.

La expansión del comercio y del número de inversiones en las que un mercader podía estar inmerso a un mismo tiempo creó la necesidad de una integración y un ordenamiento que, en cierto modo, la Partida Doble exigía y propagaba. Un comercio más amplio demandaba un sistema gerencial mejor. Ese buen sistema gerencial -la partida doble-, fue un factor decisivo en el incremento comercial.

Palabras clave: mercaderes; factores; Partida Doble; comercio. era, some double-entry concepts are dealt with. The study concludes that double-entry was at the same time cause and effect of the transition from travelling merchants to resident ones.

The expansion of commerce and the number of investments a merchant could be involved in at a time created the need of an integration and an order that, to certain extent, double-entry implied and spread. A bigger commerce demanded a better management system. This managerial system -the double-entry-, was a conclusive factor in the commercial growth.

Key words: merchants; factors; double-entry; commerce.

Mucho es lo que se ha escrito sobre la aportación de la partida doble al desarrollo económico de Occidente. El embrión de esta polémica intelectual lo encontramos en la obra de Werner Sombart Der moderne Kapitalismus, que el autor alemán comenzó a publicar en 1902 y que concluyó en $1916^{1}$. En una visión, a nuestro juicio limitada en exceso, los defensores han revestido la partida doble con una aureola dorada, y exagerada, mientras que los detractores sólo han visto en ella una técnica que únicamente ha desempeñado un papel discreto. Lo cierto es que no ha sido ni lo uno ni lo otro. La polémica se ha suscitado entre historiadores económicos e historiadores contables. Y puede que ahí radique el problema. Una visión equilibrada y en perspectiva no puede disociar la historia económica de la historia de la contabilidad, como si fueran dos campos de estudio diferentes. Ambas historias no discurren por líneas paralelas, pues transitan por la misma senda; han de manejar los mismos acontecimientos y extraer conclusiones que arrojen luz sobre la sociedad que, en cada

1 Para este estudio hemos manejado la edición de Werner Sombart, Der moderne Kapitalismus. Historisch-systematische Darstellung des gesamteuropäischen Wirtschaftslebens von seinem Anfängen bis zur Gegenwart, München und Leipzig: Verlag von Duncker \& Humblot, 1928. 
momento, se ha servido de los mejores medios a su alcance para lograr un mayor grado de desarrollo, de riqueza, de progreso y de bienestar².

Así, el Profesor Yamey ha señalado que:

Werner Sombart was largely responsible for the broad thesis that systematic or scientific accounting, identified with the double-entry system, played an important part in realising, activating, stimulating or accentuating the "rationalistic pursuit of unlimited profits," an essential element in the capitalistic spirit ${ }^{3}$.

Ese mismo historiador contable ha expresado sus dudas sobre la importancia de la partida doble en el proceso de transformación entre 1200 y 1400 , del mercader viajero o itinerante en mercader residente o sedentario ${ }^{4}$ y ha manifestado su escepticismo sobre la vinculación de la partida doble con la búsqueda del beneficio condensándolo en tres conceptos: el cálculo de la cuota de beneficio mediante cuentas de balance, de pérdidas y ganancias y de capital en los ejemplos más tempranos de partida doble es poco frecuente; el grado de dependencia de esos cálculos de las cuentas que no estuvieran en partida doble y hasta qué punto esos cálculos pudieron hacerse sin la ayuda de la partida doble. De modo similar, por lo que se refiere al control que los mercaderes sedentarios pudieron ejercer sobre sus factores en el extranjero, ha afirmado que la contribución de la partida doble fue ciertamente modesta. No cabe duda de que, desde esta perspectiva, lo dicho a principios del siglo XX por Werner Sombart no tendría demasiada importancia para la historiografía contable. Esta deficiencia, en parte, es achacable a la falta de precisión en el uso del término partida doble. Se requiere, pues, un análisis detallado del papel que desempeñó la contabilidad en la génesis del capitalismo. Dejando a un lado la conexión psicológica con el espíritu del capitalismo, la contabilidad, como herramienta de gestión, fue un factor clave en la organización comercial.

2 Cfr. B.S. Yamey, "Scientific Bookkeeping and the Rise of Capitlism," Economic History Review, 1, 1949, pp. 99-113; "Accounting and the Rise of Capitalism: Further Notes on a Theme by Sombart," Journal of Accounting Research, 2, 1964, pp. 117-136 (inicialmente se publicó en Studi in Onore di Amintore Fanfani, Milan: Dott. A. Giuffre, 1962, vol. VI, pp. 833-57); "Notes on Double-Entry Bookkeeping and Economic Progress," Journal of European Economic History, IV, 3 (Winter, 1975), pp. 717-723; F.C. Lane, "Double Entry Bookkeeping and Resident Merchants," Journal of European Economic History, 6 (1977), pp. 177-191. by Sombart," ed. cit., p. 117

B.S. Yamey, "Accounting and the Rise of Capitalism: Further Notes on a Theme

4 B.S. Yamey, "Notes on Double-Entry Bookkeeping and Econmic Progress," ed. cit. 
Uno de los objetivos que persigue el presente estudio es, precisamente, analizar esa función.

\author{
II
}

Todos los especialistas reconocen que en el período 12001400 se produjeron cambios importantes en la metodología comercial y en la organización de los negocios. El Profesor Raymond de Roover los tipifica como la Revolución comercial del siglo XIII. Y, a pesar de que este término se ha aplicado habitualmente a la expansión transoceánica del comercio que precedió a la Revolución industrial, y en la actualidad se aplica, como hace el Profesor Robert S. López, a los profundos cambios sociales que se produjeron en la Europa Occidental con la expansión del comercio en los siglos XI y XII se produjeron a finales del siglo XIII y principios del XIV, siempre y cuando se utilice con la aclaración de que la revolución a la que se refiere no fue un cambio en la estructura social, como el que se produjo en la Europa Occidental de los siglos XI y XII, sino una revolución en la práctica comercial. Supuso un cambio en la organización mercantil, de la que el Profesor N.S.B. Gras destaca el surgimiento del capitalismo comercial derivado del pequeño capitalismo y la sustitución del mercader itinerante por el sedentario:

Sedentary merchants organized the existing facilities of production and marketing into a larger pattern, in which importing, exporting, and wholesaling constituted the key or center. In a general way, this meant that favourably located towns would grow into larger centers and a few, later, into metropolitan cities. This meant that just as petty capitalists became subordinated to larger towns. Hand in hand with this process came the gradual extension of trade in large volume to most distant seas; finally, through discovery and exploration, completely around the world ${ }^{6}$.

5 Cfr. Robert S. Lopez, "The Trade of Medieval Europe: The South," en The Cambridge Economic History of Europe, vol. II, Trade and Industry in the Middle Ages, Ed. by M.M. Postan and Edward Miller, Cambridge University Press, 1987, p. 389 y del mismo autor The Commercial Revolution of the Middle Ages, 950-1350, Cambridge University Press, 1976.

6 N.S.B. Gras, "Capitalism -Concepts and History" en Frederic C. Lane and Jelle C. Riemersma, eds., Enterprise and Secular Change. Readings in Economic History, London: George Allen and Unwin Ltd., 1953, p. 74. 
Benjamin Kedar lo refleja de manera gráfica en el capítulo "From the Poop to the Desk: the Reorganization of Commerce" ("De la popa al escritorio: la reorganización de comercio") de su libro Merchants in Crisis: Genoese and Venetian Men of Affairs and the FourteenthCentury Depression ${ }^{7}$. Y el Profesor de Roover resume el concepto de forma nítida:

By a commercial revolution I understand a complete or drastic change in the methods of doing business or in the organization of business enterprise just as an industrial revolution means a complete change in the methods of production, for example, the introduction of powerdriven machinery. The commercial revolution marks the beginning of mercantile or commercial capitalism, while the industrial revolution marks the end of $\mathrm{it}^{8}$.

El Profesor Renouard, a su vez, señala:

Les hommes d'affaires qui se sédentarisent ainsi dans les villes adoptent tout naturellement de nouvelles méthodes de travail que leur proposent d'ailleurs les progrès constants de la technique. Ils perdent moins de temps et ne s'épuisent plus aux fatigues de la route et de la mer. Ils ont donc plus de facilitẻ pour entreprendre simultanément des affaires de types et de caractères divers. Et ils le font d'autant mieux que l'invention de la comptabilité à partie double vient leur permettre de dominer l'ensemble de leurs activités. L'homme d'affaires sédentaire peut être un industriel qui, par le système de l'entreprise capitaliste, dirige la fabrication ou l'affinage de draps par une série de travailleurs specialisés appartenant à une dizaine ou une vingtaine de corporations différentes et qui dépendent tout de lui en définitive?.

Pero volvamos al Profesor Raymond de Roover. Menciona cinco aspectos que generaron la nueva organización comercial. Una de ellas fue una teneduría de libros perfeccionada:

7 Benjamin Z. Kedar, Merchants in Crisis: Genoese and Venetian Men of Affairs and the Fourteenth-Century Depression, New Haven and London: Yale University Press, 1976, pp. 21. 42.

8 Raymond de Roover, "The Commercial Revolution of the Thirteenth Century" en Frederic C. Lane and Jelle C. Rimersma, eds., Enterprise and Secular Change. Readings in Economic History, ed. cit., pp. 80-85.

9 Yves Renouard, Les hommes d'affaires italiens du Moyen Âge, Paris: Librairie Armand Colin, 1949, pp. 174-175. 
(1) Instead of forming partnerships for the duration of a single venture, a new type of partnership agreement was evolved: the terminal or permanent partnership....

(2) The "letter of payment," or bill of exchange, was developed to meet the need for a more practical instrument. It made possible the transfer of money from place to place without the shipping of actual coins.

(3) The greater security along the roads....

(4) The development of maritime insurance....

(5) The bookkeeping of the travelling merchant had been crude though adequate for his purpose....

In the late thirteenth and early fourteenth centuries, accounting advanced with great strides. One innovation of major importance was the current account kept in bilateral form, that is, the personal account divided vertically into two columns (...). Later, double-entry bookkeeping was introduced by adding impersonal accounts to the existing personal accounts. Good methods of bookkeeping were essential in order to keep accounts straight when two persons, residing in different cities, had numerous business dealings with each other. Merchants had to know where they stood, and accounting served as a guide by revealing profits and losses ${ }^{10}$.

En la última parte de este estudio analizamos si los good methods que señala de Roover eran una verdadera partida doble; si este fuera el caso, habremos de ver si fue la partida doble la que hizo que los métodos utilizados fueran buenos. Pero a fin de que no dé la sensación de que nuestra exposición se escuda en la terminología técnica es pertinente que nos centremos en la manera que los mercaderes tenían de llevar y utilizar sus cuentas.

III

La contabilidad que podemos contemplar en los libros de los mercaderes venecianos de principios del siglo $X V$ se denomina contabilidad de operaciones, pues muestra la pérdida o la ganancia de cada inversión o negocio de forma individualizada. Registra las

10 Raymond de Roover, "The Commercial Revolution of the Thirteenth Century," ed. cit., pp. 81-82. La itálica es nuestra. 
inversiones comerciales mediante cuentas de mercaderías en las que se carga la mercancía comprada o recibida de los factores en el extranjero; registra, igualmente, las operaciones financieras en letras de cambio mediante cuentas de cambio. Además utiliza cuentas de viaje o embarque en las que se carga la mercancía que se envía a un lugar o factor determinados, abonándose la misma cantidad en la correspondiente cuenta de mercaderías. La suma del envío se abona en la cuenta de viaje y se carga en la cuenta personal del factor. Las cuentas de viaje se cierran en una de pérdidas y ganancias. La cuenta personal del factor se cierra abonándole por las remesas del viaje de vuelta. El Profesor Lane señala al respecto:

The venture system of accounting used at Venice was the most practical form for merchants much of whose wealth was coming on the seas. It could be varied to suit many situations as is shown by the extant Venetian account books, few as they are. It was a flexible system which enabled a merchant, while keeping a clear and accurate record of his obligation and his debtors, to calculate not regularly but easily and realistically his profits and losses ${ }^{11}$.

Este sistema operativo de teneduría de libros por partida doble es el fundamento del valor que se ha de conceder a la partida doble, tal y como señala el ya mencionado Prof. Lane:

The use of resident agents instead of travelling merchants was facilitated by a number of improvements in commercial technique. One was the system of doubleentry bookkeeping. This way of grouping and checking the records of every transaction made it easier for a resident merchant to keep track accurately of what his partners or agents were doing (...). This kind of bookkeeping enabled a merchant operating simultaneously on many market places to know the extent and nature of his assets $^{12}$.

En esta misma línea están las ideas de Hermann Kellebenz:

The value of double-entry bookkeeping for the day to day Management and control of a firm's assets was

11 Frederic C. Lane, Venice and History: The Collected Papers of Frederic C. Lane, Baltimore: The Johns Hopkins Press, 1966, p. 108.

12 Frederic C. Lane, Venice, A Maritime Republic, Baltimore: John Hopkins University Press, 1973, pp. 140-141. 
simply that it made easier to marshal and keep an eye on facts relevant to running the business. And the new system certainly made it easier for a business like the Fuggers to control their network of agents than it had been, let us say for the Veckinghusens, a century earlier with a less highly developed system. Subsequent to this development, which continued into the sixteenth century, and the further expansion of the new Italian system by way of Antwerp, London and Hamburg, nothing was added until the eighteenth century ${ }^{13}$.

El Profesor Yamey disiente de la postura de Lane y Kellebenz y argumenta que ningún sistema de teneduría de libros puede facilitar al mercader información fidedigna de lo que hacen sus socios o factores, pues depende de estos decidir cómo disponen de los activos que les ha encomendado, cómo se hallan las deudas pendientes y de qué modo adquieren activos en su nombre ${ }^{14}$.

Debemos admitir que un mercader residente en Venecia dependía de su factor en el extranjero para saber a qué precio se había vendido la mercancía enviada y el pagado por los productos recibidos a cambio, pero al disponer de una cuenta saldada por el factor con cantidades precisas para concepto de gasto y su correspondiente recibo, podía cotejar sus datos con los informes de su factor y los de otros factores; todo este proceso generaba un flujo constante de correspondencia que hacía que el mercader veneciano estuviera "sufficiently well informed about prices so that he could send instructions to his agents and check up afterwards to see how advantageous his agent had traded for him"15. Tampoco podemos olvidar los manuales de mercaderes que informaban sobre sus precios habituales. La contabilidad de operaciones no impedía que un factor o socio informara indebidamente de los precios y gastos; del mismo modo que en la actualidad nada impide que se falsifiquen los inventarios. Los censores jurados de cuentas no inspeccionan las bodegas de los barcos ni examinan si las toneladas de petróleo que entran o salen de un puerto, sólo por poner un ejemplo, coinciden con las detalladas en las respectivas

13 Hermann Kellebenz, "Technology in the Age of Scientific Revolution 15001700," en Carlo M. Cipolla, ed., The Fontana Economic History of Europe. The Sixteenth and Seventeenth Centuries, Glasgow: William Collins Sons \& Ltd., 1974, vol. 2, p. 234.

cit., p. 718.

14 B.S. Yamey, "Notes on Double-Entry Bookkeeping and Economic Progress," ed.

15 Frederic C. Lane, Venice, A Maritime Republic, ed. cit., p. 140. 
cuentas. Es cierto; pero este detalle no demuestra que la contabilidad no aporte nada a la gestión de los negocios del pasado y del presente.

La contabilidad no sólo puede informar sobre errores y omisiones matemáticas; también puede mostrar discrepancias, inconsistencias y falsificaciones. La contabilidad de operaciones utilizada en Venecia en el siglo XV cumplió estos objetivos; como también lo hizo la contabilidad de sociedades toscana. Todo lo dicho hasta ahora no es obstáculo para estar de acuerdo con lo señalado por el Profesor Yamey: "The efficacy of the merchant's control over his distant factors depends, in turn, on his success in the choice of factors, the training he gives them and the sanctions he is able to apply ex post facto" ${ }^{16}$.

No obstante, tomando estas palabras como referencia, podemos observar que la contabilidad veneciana sirvió para formar factores, seleccionarlos y sancionarlos.

Por lo que se refiere a la formación de los jóvenes mercaderes de Venecia no es mucho lo que sabemos, salvo que trabajaban bajo la supervisión de mercaderes maduros, en su mayoría parientes, que viajaban y que acudían a las Escuelas de Abaco en donde les enseñaban contabilidad y aritmética ${ }^{17}$. A juzgar por los resultados, buena parte de la práctica estilística para llevar las cuentas ya se encuentra instituida a comienzos del siglo XV. Las cuentas de viaje o embarque y las de mercaderías eran habituales y mantenían una uniformidad; donde se observan más variaciones es en las cuentas de pérdidas y ganancias, las de gastos y las de ingresos acumulados. Sin embargo, las similitudes entre los Libros Mayores de Andrea Barbarigo, Jacomo Badoer y otros mercaderes venecianos de principios del siglo XV apuntan un alto grado de uniformidad, fundamentalmente en las cuentas más útiles para tratar con los factores: cuentas de viaje, que registran las remesas enviadas; cuentas de mercaderías; los envíos recibidos y las cuentas personales de los factores o socios que reciben o envían la mercancía. El Profesor de Roover dice al respecto:

Typical of Venetian accounting are the venture or voyage accounts, which have not disappeared to this day as they are still currently used by shipping companies.

cit., p. 718.

16 B.S. Yamey, "Notes on Double-Entry Bookkeeping and Economic Progress," ed.

17 Sobre la educación de los mercaderes véase Armando Sapori, Studi di Storia Economica Medievale, Firenze: G.C. Sansoni, 1946, pp. 285-325. 
These accounts were charged with the value of any goods "recommended" to the care of agents abroad and with any expenses incurred thereon. To offset these charges, such accounts were credited with the proceeds of the sales as detailed in the agent's report. In the trade with the Levant, it rarely happened that "returns" were made in cash or in bills of exchange. As a rule they were made in eastern wares for which the agent received credit; to this credit there usually correspond a charge to a newly opened merchandise account, which was not closed until each lot was fully sold (...). Both voyage and merchandise accounts were closed into Profit and Loss after each affair was completely finished. Since agents sometimes failed to report or goods were slow in moving, it happened that such accounts remained in abeyance for months or even years. It was one of the serious drawbacks of the Venetian system ${ }^{18}$.

Debe tenerse en cuenta que, por lo que se refiere a la forma contable utilizada para anotar las remesas enviadas o recibidas, no se aprecian diferencias derivadas de que el consignatario sea un socio con plenos poderes, un trabajador a sueldo o un factor a comisión. La contabilidad de operaciones estaba preparada para ocuparse de los factores a comisión que, por lo general, recibían un dos por ciento de las ventas y un uno por ciento de las compras $^{19}$. En los Libros Mayores de Barbarigo y Badoer las cuentas de los factores son un elemento importante, en concreto en el de Badoer, que registra su propia actividad como factor. Estos Libros Mayores demuestran que era habitual que el principal de una operación se convertía en factor de otra. En el círculo veneciano al que ambos pertenecían existía un lenguaje de la contabilidad bien conocido. Este aspecto no es menor si consideramos que los mercaderes ingleses del siglo XVI no disponían de una herramienta tan importante como es la utilización de un mismo lenguaje contable.

18 Raymond de Roover, "The Development of Accounting Prior to Luca Pacioli According to the Account Books of Medieval Merchants" en Raymond de Roover, Business, Banking and Economic Thought in Late Medieval and Early Modern Europe, Ed. by Julius Kirschner, University Press of Chicago, 1974, pp. 161-164. Para observar fragmentos y explicaciones finísimas de cuentas del siglo XV veneciano, véase la obra monumental de Federigo Mellis Documenti per la storia economica dei secoli XIII-XVI, con una nota di Paleografia Commerciali a cura di Elena Cecchi, Istituto Internazionale di Storia Economica "F. Datini", Prato. Publicazioni-Serie I, Documenti, Firenzi: Leo S. Olschki, 1972, Documentos 137 y 138.

19 J. Lanero Fernández y E. Ortega Montes, "De aprendiz a mercader: El factor en el comercio internacional inglés del siglo XVI", Pecvnia, 5 (2007), pp. 145-180. 
Todos sabían lo que se debía recoger en los libros de alguien con quien se realizaba una operación. Ambas partes utilizaban la disposición de cargos y abonos en columnas paralelas: los cargos a la izquierda y los abonos a la derecha; la dualidad de las partidas queda clara por las referencias cruzadas al correspondiente cargo o abono de cada partida. Se utiliza la contabilidad de operaciones. Las partidas dobles que se manejan para saldar las cuentas hacen posible la incorporación de los resultados, provistos por los factores, en el sistema contable usado en el Libro Mayor del principal. Cuando se trata de mercancía para un corresponsal, el consignatario abre una cuenta de mercaderías por cada remesa recibida en la que abona las cantidades de las ventas, carga los gastos y la salda cargándole un trato neto. El factor anota el trato neto como abono en la cuenta de quien, o a nombre de quien, se recibe la remesa, en caso de que no existan otras indicaciones. Entra dentro de las obligaciones del factor enviar al principal una copia de la cuenta de mercaderías, según se indica en el tratado de Ympyn, de clara inspiración pacioliana, en su traducción inglesa de 1547:

And if it fortune any debtor whiche hath had long to doo with you, to come and desire you to make hym a copie of his reconnying out of your boke, whiche you maie not well saie nay, then shall you take a leafe of paper as great as the reconnyng doth require, and rule it after the maner of the greate boke [the ledger], ... ${ }^{20}$.

El principal, a su vez, abona en su cuenta de viaje o embarque la cantidad del trato neto y carga al factor la misma cantidad. Los detalles de las cuentas de mercaderías que el factor abre por las remesas recibidas suministran al principal una información valiosa que le pueden ayudar a ver si ha elegido acertadamente a su factor o debe buscarse otro.

Tomemos un ejemplo: Jacomo Badoer abre en Constantinopla, en 1437, una cuenta de Vino de Messina para anotar 160 de 169 barriles recibidos (nueve le correspondían por comisión). Una cuarta parte era del patrón del barco, Todoro Vatazi de Messina, y las otras tres cuartas partes de Piero Michiel y Marin Barbo de Venecia. El

Progress," ed. cit., p. 723.

20 Citado en B.S. Yamey, "Notes on Double-Entry Bookkeeping and Economic 
remitente de la remesa era Nofrío da Chalzi ${ }^{21}$. Badoer residió en Constantinopla durante tres años (1436-1439) para ocuparse de sus negocios y los de su hermano, que continuó residiendo en Venecia:

Giacomo Badoer si reca a Constantinopoli per curare gli affari di commercio propî e del fratello Girolamo, che si trattiene invece a Venezia, e si mantiene con lui in continua corrispondenza, gli spedisce e ne riceve le merci, e di tratto in tratto gli fornisce qualche somma di denaro per mezzo di lettere di cambio. Egli tratta, spesso in misura considerevole, affari suoi personale e più spesso in società, a parte uguali, col fratello, ma nello stesso tempo trata anche affairi di altri patrizi veneziani, fra i quaili sono in primissima linea Pietro Soranzo e Pietro Michiel, di cui l'attività è ininterotta e spesso assai relevante per tutti i tre anni ${ }^{22}$.

Pero volvamos a la cuenta de Badoer. Las siete primeras partidas del Debe, fechadas el 20 de agosto de 1437, se refieren a gastos que los principales, Piero Michel y Marin Barbo, se entiende que mercaderes residentes en Venecia, podían comprobar: el impuesto por barril pagadero al Bailo; gasto del transporte al almacén; honorarios de un catador de vinos por la venta; honorarios del factor; el flete por barril; impuestos del emperador griego; comisión de Badoer, en la que incluye el salario pagado a un mozo de almacén. Hay que poner en duda que Badoer pagara esas cantidades, pues hay constancia en sus libros de que en ocasiones saldaba las deudas con los acreedores mediante otros medios, como dándoles vino de mala calidad a bajo precio. Sea como fuere, los gastos relacionados eran los que contemplaba la ley o la costumbre, y que los mercaderes residentes en Venecia conocían bien por un intercambio constante de correspondencia y por los manuales de mercaderes de la época. El de Francesco Balducci Pegolotti, La pratica della mercatura, es el más conocido (1340; 1471):

21 Giacomo Badoer, El libro dei conti di Giacomo Badoer (Constantinopoli, 14361440), testo a cura di Umberto Dorini e Tommaso Bertelè, Roma: Librería dello Stato, 1956, cartas $98,132$.

22 Gino Luzzatto, Storia economica di Venezia dall'XI al XVI secolo, Venezia: Centro Internazionale delle Arti e del Costume, 1961, p. 172. 
Francesco Balducci Pegolotti, La pratica della mercatura (principios del siglo XIV) Biblioteca Riccardiana (Florencia) Manuscrito 2441, fol.1 ${ }^{\mathrm{r}}$

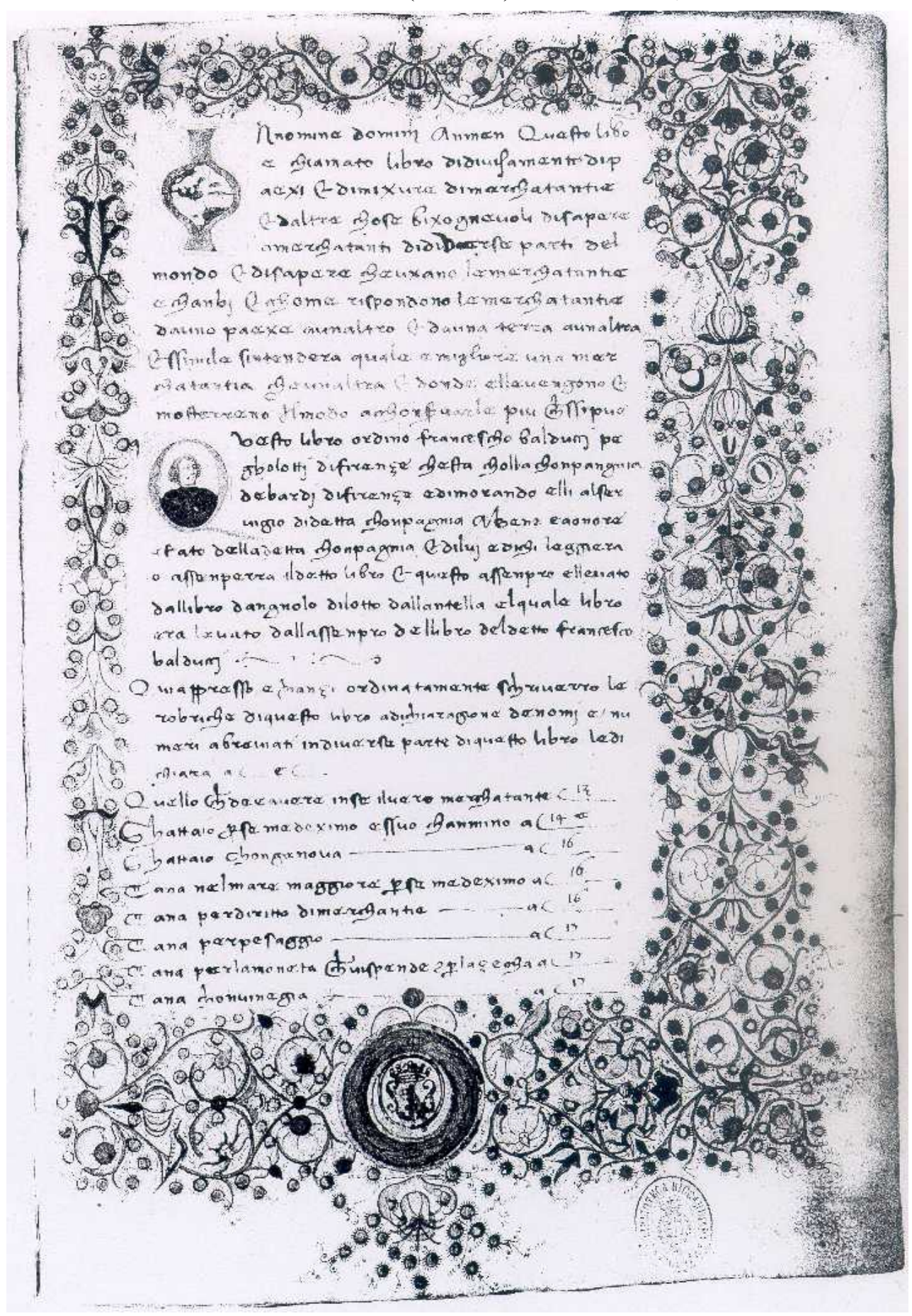




\section{Constantinopoli e Pera per ispese che si fanno di vino di ponente}

Primeramente, per portare il vino dalla marina al magazzino e per istivarlo nel magazzino, da carati $1 \frac{1}{2}$ in 2 per botte.

Per loghiera di magazzino ne puote venire per una botte da carati $1 / 2$ in 1 il mese.

E per farlo gustare, e vedere le botte como sono sceme, da carati 6 per 100 bolti.

E per farlo vedere á cernitori che ne traggono fuori quello che dè essere recuso, da carati 6 in 12 per 100 di botti.

E quando vendi, il venditore si'l fa trarre fuori del magazzino le botti, e se alle teste delle botti mancha cerchi che mancano nelle teste si gli dee rifare il venditore al comperatore; ma se $\mathrm{i}$ cerchi delle teste sono alle botti e tutti gli altri cerchi ne fussino fuori, non è tenuto il venditore di fare niuno cerchio al comperatore. E puossi ragionare tratarle del magazzino e facimento di cerchi in somma da carato 1 per botte ${ }^{23}$.

Además del de Pegolotti, existieron manuales en Toscana, Génova y Venecia, aunque los ejemplares más tempranos son venecianos ${ }^{24}$.

En el ejemplo que estamos viendo de Badoer, los gastos de flete de Messina a Constantinopla eran variables, aunque en este caso se pueden cotejar por el patrón del barco que tenía negocios con Piero Michiel y Marin Barbo.

Los abonos en esta cuenta de Vino de Messina, del 28 de agosto al 4 de noviembre, confirman que se distribuyó en veintidós ventas de menor cuantía y una grande de ochenta y siete barriles; en cada caso se especifica la cantidad, el precio por zentener y varias formas de pago. Un par de partidas corresponden a unos barriles de vino estropeado. Los abonos ascienden a 2867 peperi, 3 karati. Una vez deducidos los cargos por los conceptos mencionados, 120 peperi, 19 karati, restan 1656 peperi, 8 karati, que es el trato neto, una vez saldada la cuenta del vino.

23 Francesco Balducci Pegolotti, La pratica della mercatura, Ed. by Allan Evans, Cambridge, Massachusetts: The Medieval Academy of America, 1936, p. 47.

24 Ugo Tucci, "Tariffe veneziane e libri toscani di mercatura", Studi veneziani, X (1968), pp. 65-108. 
Tres cuartas partes de este trato neto del vino se anotan como abono en la cuenta de un mercader de Messina, no en la de Piero Michiel y Marin Barbo, según las instrucciones que Badoer recibió de éstos, para que efectuara pagos en su nombre. La otra cuarta parte se pagó de igual forma, siguiendo las indicaciones de patrón del barco, Todaro Vatazi. En ambos casos los abonos en esas cuentas se hacen contra los cargos de otras operaciones. Por ejemplo: en los libros de Badoer se carga al mercader en Messina, Nofrio da Chalzi por remesas de pimienta y transporte de esclavos.

Antes de examinar la utilidad de la contabilidad de operaciones veneciana para seguir el hilo del saldo, veamos cómo la cuenta de mercaderías que llevaba Badoer como factor a comisión servía para que un mercader en Venecia controlara a su factor en el extranjero. Después de recibir durante un par de años las cuentas de beneficios de sus envíos y el coste de las remesas de vuelta, el mercader residente podía comprobar la aritmética del factor; si había añadido otros gastos fuera de lo habitual y no incluidos en los manuales; si los precios de venta o de compra estaban por encima o por debajo de los otros comerciantes en ese mismo momento y lugar; si el flete correspondía a la cantidad que se había pagado. No es anecdótico que en un envío de Constantinopla a Beirut, a bordo de un barco cretense, Giacomo Badoer fuera capaz de localizar un cargo indebido que le notificó un corresponsal en Beirut ${ }^{25}$.Y aún cuando el consignatario pagara el flete, el consignador podía conocer la cantidad pagada bien por haber negociado con el patrón del barco en el que había cargado por haber oído las tasas que otros consignadores pagaban. Muchos envíos pertenecían a varios propietarios, por lo que las tarifas eran bien conocidas.

Incluso cuando las discrepancias fueran de carácter menor, podían llevar al mercader residente a tomar la decisión de continuar con ese factor o buscarse otro, o de renovar o no la sociedad. Si encontraba serios errores o inconsistencias en los informes del factor, o entre estos y las noticias que le llegaban del mercado de Rialto o por las cartas de sus corresponsales, podía llevar al factor a los tribunales. En ese caso, los libros de cuentas se convertían en una parte fundamental de las pruebas. El factor podía ser llamado para que presentase sus libros para desmontar las acusaciones presentadas contra él.

25 Giacomo Badoer, El libro dei conti di Giacomo Badoer (Constantinopoli, 1436- 
IV

Los libros y correspondencia de Andrea Barbarigo nos muestran cómo un mercader residente en Venecia podía comprobar lo que hacían los factores residentes en el extranjero con sus comisiones. Cuando se trataba de procesos judiciales, la correspondencia y las cuentas servían para argumentar el caso; y aunque el mercader no acudiera a los tribunales, su disconformidad con el factor, que se basaba en la elección de las opciones de éste dentro del marco legal, si bien de manera que no satisfacían los beneficios que Barbarigo esperaba obtener, provocaron que el mercader analizara aspectos similares a los ya vistos en la cuenta de vino de Badoer. Barbarigo acusó a su factor, que compraba algodón en Palestina, de exagerar las cuotas pagadas al consulado; de no especificar la medida total del paño que le había enviado; de no indicar el peso exacto de las remesas enviadas y hacer deducciones por deterioro o pérdida, aunque no se hubieran producido. Igualmente comprobó los precios que el factor le había detallado en cartas anteriores con los muy superiores que sus cuentas mostraban que había pagado por el algodón. Las cuentas tan solo constituían un elemento en este tipo de comprobaciones, pero eran una contribución muy notable:

Among the methods of proof accepted by the law court were the letters received and the accounts rendered. They were more convincing than oral testimony. A merchant could be called on to submit his account books to the examination of the judges and was expected, even if he had no formal receipt, to have letters to show. Accounts and letters were basic to legal action for enforcing the responsibility of the overseas agents to the merchants resident in Venice ${ }^{26}$.

La forma de organizar las cuentas también era importante para seguir la pista del pago de beneficios. Dicho en otras palabras: para seguir el hilo de todas las obligaciones que se hubieran contraído, es decir: todos los abonos y cargos generados en cuentas personales por el conjunto total de las operaciones. El pago de beneficios en muchos casos no es fácil de identificar. El pago, con frecuencia, consistía en abonar a alguien con el que el principal estaba en deuda. El Profesor Lane lo ha demostrado haciendo un análisis exhaustivo de las cuentas de Andrea

26 Frederic C. Lane, Andrea Barbarigo, Merchant of Venice, 1418-1449, Baltimore: The Johns Hopkins Press, 1944, p. 98. 
Barbarigo durante el bienio 1441-1443 referentes a su negocio de paños en Inglaterra. Debemos tener presente que:

[Barbarigo] was not a rich man in comparison with many other Venetian nobles, but he had begun with next to nothing and in 1440 disposed of a capital of about 10.000 ducats. Almost most of this was in commerce, practically none in government bonds or real estate. He was a merchant who diversified his commercial investments very widely, traded in many wares and to many countries, and sold both by wholesale and, through agents, at retail. The branch of his affairs to which he gave most personal attention, aside from bills of exchange, was the import and resale of English cloth ${ }^{27}$.

Las dudas, dada la diversificación de los negocios, se podían originar en operaciones absolutamente independientes unas de otras. Puede que fueran negocios conjuntos (joint ventures) con terceras, cuartas y quintas partes. En el caso concreto del vino que Badoer vendió como factor a comisión, tres cuartas partes de su pago adoptaron la forma de un abono en la cuenta de Nofrio da Chalzi. Una partida importante en el Debe de la cuenta de Chalzi se refiere al coste del envío de una remesa de cobre que Badoer realizó siguiendo sus instrucciones. El cobre era para Luca y Andrea Vendramin, Doge de Venecia de 1476 a 1478, aunque Badoer desconocía el motivo ("de chi raxion el sia io non 'l so" $)^{28}$. Otras partidas de los libros de Badoer muestran que Chalzi participaba en numerosos negocios conjuntos con Piero Michiel y Marin Barbo. Al mismo tiempo, el hermano de Giacomo, Jeronimo, mercader residente en Venecia, estaba invirtiendo en su nombre en la "Chonpagnia que ho fata con miser Piero Michiel e ser Marin Barbo e Jeronimo mio fradelo"29. Para cualquier mercader que hiciera negocios en este entramado laberíntico, asentar un cargo o un abono por cada operación, con referencias cruzadas entre sí, debió de ser de gran ayuda para seguir el hilo conductor de sus obligaciones y cuentas pendientes de cobro.

\footnotetext{
Lane, ed. cit., p. 118

Frederic C. Lane, Venice and History. The Collected Papers of Frederic C.

28 Cfr. Giacomo Badoer, Il libro dei conti di Giacomo Badoer (Constantinopoli, 1436-1446), ed. cit., carta 142.

29 Ibid., carta 164.
} 
Conto di lana del quaderno di Andrea Barbarigo di Venezia, 1438

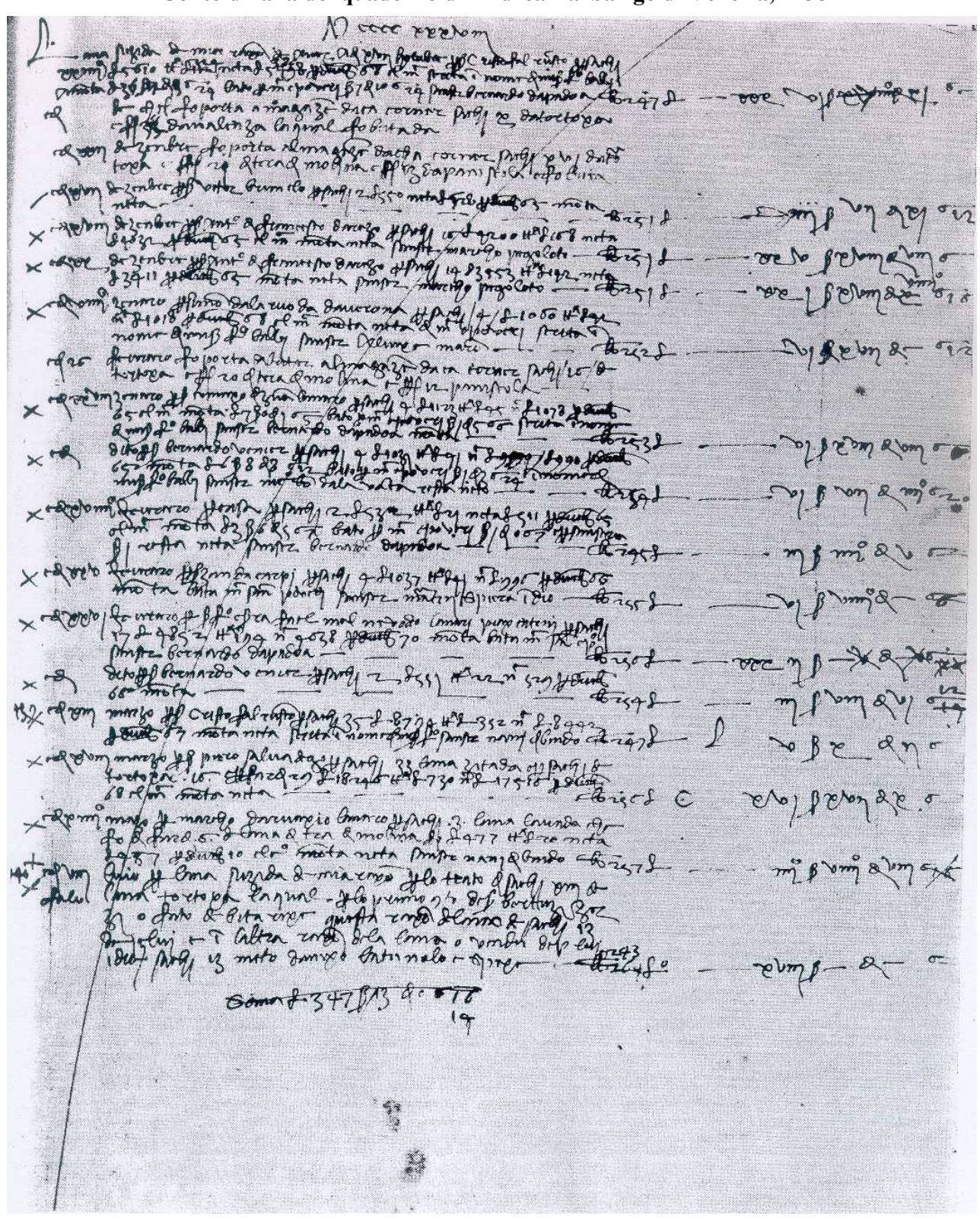

Es posible que los párrafos anteriores sirvan para comprender el grado de sofisticación que poseía el comercio veneciano 
del siglo XV; no obstante, no resulta descabellado que nos preguntemos: ¿qué tiene que ver toda esta estructura con la partida doble? La respuesta depende de lo que entendamos por partida doble. El abanico de definiciones es variado. La de Federico Mellis parte de la existencia de una empresa lucrativa independiente en la que los beneficios y activos no tienen nada que ver con los ingresos o activos de las personas, a nivel particular, participantes en el negocio:

Esposte le radici della "partita doppia", mi sento autorizzato a definirla come il metodo contabile che si fonda sulla duplice consideratione -analitica e unitariadella ricchezza agisce nell'azienda, da cui scaturiscono due segni di conti, nei quali ogni operazione si registra invariabilmente in partite di conti di segno antitetici "dare" e "avere" e dai valori eguali. A questa armonia fra serie di conti, loro partite e valori, si è pervenuti quando accanto ai preesistenti conti che contemplavano analiticamente la richezza si è affiancato il conto di capitale e quelli di "disavanzi e avanzi": ai quali ultimi, infatti, venivano imputate le variazioni esuberanti in toto o parzialmente, nell'incessante movimiento degli elementi della ricchezza, ristabilendo l'equilibrio -come in una equazione matematica- fra il "dare" e l'avere" della prima serie di conti. Ricorrendo ancora alla matematica, si può, dunque, affermare che condizione necessaria e sufficiente affinchè un registro sia improntato alla partita doppia è che in esso abbiano preso consistenza i conti di "disavanzi e avanzi", a prescindere da quello di capitale. Naturalmente, la serie di questi conti derivati deve essere completa, nel senso che essa metta in evidenza tutte le possibili variazioni di reddito. Ma è anche indispensabile, ritornando alla prima serie, che la ricchezza sia osservata in tutti i suoi aspetti: e non comme avviene nei registri di Genova, Milano e Venezia fino ad oggi ritenuti esemplari perfetti di partita doppia, e che avrebbero preçeduto di lungo tempo quelli toscani per tale prerogativa ${ }^{30}$.

El Profesor Mellis no considera esencial la disposición de las partidas, aunque estima que es fundamental un conjunto de cuentas que registre todos los cambios de valor de las posesiones de la empresa, incluidos los cambios en el valor de su equipamiento. A su modo de ver, no es necesaria una cuenta de capital siempre y cuando que la cuenta de

30 Federigo Mellis, Documenti per la storia economica dei secoli XIII-XVI, ed. cit., pp. 57-58. 
pérdidas y ganancias se utilice de forma sistemática y en combinación con otras cuentas a fin de que facilite el balance que ocuparía el lugar de la cuenta de capital par mostrar el aumento o disminución de activos durante un tiempo determinado. Es absolutamente interesante su teoría del nacimiento de la partida doble: el binomio partida doble-empresa capitalista:

La partita doppia nasce, quindi, con il concretarsi dell'impresa capitalistica e tra i due fenomeni si può parlare di correspondenza biunivoca: nel senso che l'azienda in cui si incontra la partita doppia è da ritenersi un'impresa capitalistica; e, viceversa, la constatazione dell'esistenza di questa impresa rinvia all'adozione della partita doppia. Ma ciò vale soltanto alle origini, per le origini: perché passato del tempo, ed entrato, il metodo, nelle cognizione dei ragionieri -quasi certamente con il concurso dei maestri d'abaco, che ne percepirono meglio le uniformità, riducendole a regole permnente -e degli amministratori, in genere, fu applicata a qualsiasi organissmo non capitalistico (cosi, all'azienda pubblica), perdend la funzione di segnalamento di un tal carattere dell'azienda ${ }^{31}$.

En el otro extremo tenemos la visión de los académicos japoneses a los que hay que conceder el beneficio de ser observadores del problema semántico. El Profesor W. Kimura distingue entre DoubleEntry Bookkeeping y Business Bookkeeping. La primera sería una mera forma de cálculo y un concepto suprahistórico y técnico. La segunda constituiría el concepto sustancial, histórico y económico que se halla en la base de los asuntos de la empresa que quedan registrados. Detalla la distinción citando como errónea la definición dada por W.A. Paton en 1922 y que es muy similar a la de Federigo Mellis ${ }^{32}$ : "The double-entry system is any scheme of accounting which exhibits all the property and equity facts of the business enterprise and registers, at least periodically, the changes therein." El Profesor Kimura dice que no es apropiado explicar su esencia en términos económicos tales como propiedad, capital, pérdidas y beneficios. En esencia, la teneduría de libros por partida doble es únicamente una forma de calcular y registrar. Lo que la caracteriza es la división de una página en una parte izquierda (Debe) y

31 Federigo Mellis, Aspetti della vita economica medievale (Studi nell'archivio Datini di Prato), Firenze: Leo S. Olschki, 1962, p. 394.

32 W.A. Paton, Accounting Theory, New York: The Ronald Press Company, 1922. 
una derecha (Haber) y el saldo del Debe y Haber de cada cuenta. La fórmula contable es únicamente una forma de calcular y, por sí misma, no tiene ningún significado económico ${ }^{33}$. El Profesor Osamu Kojima, a su vez, al referirse a los libros de cuentas de Barbarigo que, como es sabido, se componen de un Diario A que comprende desde el 2 de enero de 1430 al 31 de agosto de 1440, con su correspondiente Libro Mayor y el Diario B, que abarca desde el 1 de septiembre de 1440 al 18 de octubre de 1449, con su Libro Mayor B, afirma:

In the account books of Barbarigo, the merchandise accounts are closed with the posting of their balances to the profit and loss accounts, and the balance of the latter is transferred to the capital account. Thus a consistent system of accounts was established. Furthermore, in his accounting system, almost all the ledger entries were through the journal. Here we find a closely coordinated conjunction between journal and ledger. Thus, there was established a basic form of the Italian accounting system of debit and credit which prevailed in European countries at that time ${ }^{34}$.

El Profesor Yamey está en la línea de Raymond de Roover y de Florence Edler de Roover. Consideran un requisito la dualidad de las partidas, relacionadas por referencias cruzadas, pero sostienen que la teneduría de libros no está en partida doble a no ser que provea el saldo de todas las cuentas. Saldar las cuentas de mercaderías requiere una cuenta de pérdidas y ganancias y saldar una cuenta de pérdidas y ganancias precisa de una cuenta de capital. Lo de menos es la denominación; lo que se necesita es que unas cuentas realicen esas funciones. El Profesor Yamey, refiriéndose al Libro Mayor de Thomas Howell (1522-1528) es breve y conciso: "It contains a large number of bilateral accounts. It falls far short of the minimum requirements of double entry in that there is no capital account, no profit-and-loss account and no consistent duality of entry for each transaction"35. Florence Edler de Roover aplica este patrón en su estudio de Andrea

33 Wasaburo Kimura, "Double-Entry Bookkeeping and Business Bookkeeping," en Osamu Kojima, ed., Historical Studies of Double Entry, Kyoto: Daigakudo Shoten Limited, 1975, pp. $259-260$

1995, p. 62.

34 Osamu Kojima, Accounting History, Nakatomigaoka, Japón: Kohko Kojima, cit., p. 722.

35 B.S. Yamey, "Notes on Double-Entry Bookkeeping and Economic Progress," ed. 
Bianchi que, de acuerdo con aquel, no utilizó la teneduría de libros por partida doble:

The ledgers and libri segreti are kept in bilateral form, that is, the debits and credits of each account are on facing pages. Usually the entries give a crossreference to another page in the ledger -in the quaderno di cassa or in one of the auxiliary books. However, there are no accounts for operating results. To determine profits, Bianchi and his partners had to go to the trouble of making a detailed inventory and then preparing a saldo or general financial statement. Profits were not determined by balancing and closing the books but by the more laborious process of deducting liabilities and initial investment from assets (receivables, cash, and inventories of raw materials, goods in process, and finished products) $)^{36}$.

La postura de Tommaso Zerbi es bastante similar, aunque destaca que la partida doble se desarrolló a partir de la forma en la que la dualidad de las partidas se organizó en la temprana contabilidad de Lombardía, que denomina sistema tabulare y que para él es un requisito indispensable. Considera que el Libro Mayor de la compañía catalana del mercader lombardo Marco Serrainerio, de 1395, es el ejemplo más antiguo que se conoce de la aplicación completa de la partida doble a los beneficios de una empresa comercial y afirma que los venecianos que anotaron en los libros de Soranzo, Barbarigo y Badoer demostraron un dominio perfecto de las técnicas de la partida doble ${ }^{37}$.

Mellis, por el contrario, considera que en esos Libros Mayores lombardos y venecianos sólo se aprecia una partida doble rudimentaria. Por su parte halla la partida doble en pleno uso en Toscana a comienzos del siglo XIV ${ }^{38}$. Los ejemplos lombardos y venecianos son, a su juicio, una partita doppia zoppicante por su incapacidad de separar los gastos domésticos de los gastos de equipamiento de la compañía y para

36 Florence Edler de Roover, "Andea Bianchi, Florentine Silk Manufacturer and Merchant in the Fifteenth Century," en William M. Bowsly, ed., Studies in Medieval and Renaissance History, Lincoln, Nebraska: University of Nebraska Press, 1966, vol III, pp. 223-285; p. 236.

37 Tommaso Zerbi, Le origini della partita doppia: Gestioni aziendali e situazioni di mercato nei secoli XIV e XV, Milan: Marzorati, 1952, pp. 42-51.

38 Federigo Mellis, Aspetti della bita economica medievale (Studi nell'Archivio Datini di Prato, ed. cit., pp. 399-400. 
registrar en sus activos el valor amortizado de ese equipamiento ${ }^{39}$. Ante esta diversidad de opiniones, es recomendable tener presente la dualidad de las partidas como componente elemental de la partida doble. El Profesor de Roover señala al respecto:

... the duality which is the principal feature of doubleentry bookkeeping is not just a formal matter, but corresponds to a fundamental reality. In view of the inner logic of the system, I do not see why it could not have evolved out of varying antecedents ${ }^{40}$.

Y en The Cambridge Economic History of Europe dice:

The adoption of bilateral form does not necessarily mean that books are in double entry. As a matter of fact, form has little importance, but there is no double entry unless certain rules are strictly observed. First of all, it is necessary that each transaction be recorded twice, once on the debit and once on the credit side (or section), so that the books will balance if correctly kept. Second, there must be a complete set of accounts, real as well as nominal, including expense and equity accounts. Third, the records must lead up to a comprehensive financial statement or balance, which shows the assets and liabilities and enables the merchant to ascertain his profit and loss. These requirements seem to have been fulfilled in the case of the Genoese records of the massari or municipal stewards $(1340)^{41}$.

Una vez iniciados en la partida doble, los mercaderes repararon en las ventajas que se podían derivar de su desarrollo. Los beneficios que se hubieran podido obtener con un sistema organizado de partida doble quedaron patentes en su evolución, que representaba los principios de un crecimiento económico. En este sentido, el Profesor Yamey ha señalado:

From the point of view of routine administration and the control of assets, the merit of the double-entry system lies in its comprehensiveness and its possibilities

\footnotetext{
39 Federigo Mellis, Docuemnti per la storia economica dei secoli XIII-XVI, ed. cit., p. 59.

40 Raymond de Roover, "New Perspectives on the History of Accounting," The Accounting Review, XXX (1955), pp. 405-420; p. 413.

${ }^{41}$ Raymond de Roover, "The Organization of Trade," en M.M. Postan, E.E. Rich and Edward Miller, eds., The Cambridge Economic History of Europe. Economic Organization and Policies in the Middle Ages, Cambridge University Press, 1963, vol. III, p. 92. La itálica es nuestra.
} 
for the orderly arrangement of data. In a sense the adoption of the system compels a certain degree of comprehensiveness and orderliness because, as every schoolboy knows, for every debit there must also be a credit. Thus data may be recorded in the system which, but for the compulsion of the system, the business man might at the time prefer to have ignored, and subsequently the availability of the additional data might prove to be useful. And orderliness of arrangement is an obvious help in routine administration ${ }^{42}$.

El mérito, pues, no reside en que la partida doble se utilizara para analizar innovaciones empresariales, sino en la administración rutinaria y en el control de activos. Los pasos necesarios que hubieron de darse hacia esa integración se hallan en la personificación de la caja y, posteriormente, de ciertas mercancías. La personificación se observa en la redacción "La caja debe dar (Cassa $d$ " dare)", "La caja debe recibir (Cassa de avere)"; posteriormente se empleó terminología como "El vino debe dar", "El vino debe haber" o "El vino ha dado". Las cuentas de mercaderías creadas por este procedimiento sólo se podían saldar mediante la aplicación de un método para calcular las pérdidas y ganancias, mientras que las cuentas personales, a su vez, se autosaldaban, a no ser que existieran errores o deudas incobrables. Las cuentas de mercaderías, pues, fueron fundamentales para hacer de la partida doble un instrumento útil para el cálculo de pérdidas y ganancias.

Que la partida doble, en efecto, se había desarrollado hasta ese punto antes de 1340 es bien conocido por las cuentas del Comune de Génova donde encontramos, a modo de ejemplo, una cuenta de pimienta saldada mediante una cuenta de pérdidas y ganancias. En esa época, las líneas de desarrollo en Toscana, por una parte, y en Lombardía y Venecia, por otra, eran divergentes. La contabilidad empresarial toscana se centró, sobre todo, en la necesidad de las sociedades, constituidas para un breve espacio de tiempo, de calcular los beneficios y distribuirlos entre los socios según estuviera estipulado en el acta de constitución de la sociedad. La contabilidad toscana distinguía entre el capital de la sociedad, como entidad abstracta, la compañía o negocio (Azienda) y el patrimonio personal de los socios. Igualmente, facilitaba el material para los balances periódicos de la compañía. Por otra parte, los libros de cuentas venecianos

42 B.S. Yamey, "Accounting and the Rise of Capitalism: Further Notes on a Theme by Sombart," ed. cit., pp. 133-134. 
perfeccionaron y extendieron la contabilidad de operaciones. A la cuenta de mercaderías le añadieron la de embarque o viaggi con el fin de poder registrar de forma más analítica los resultados de las remesas que se iban a vender a través de los factores. Desde una perspectiva veneciana se siente la tentación de identificar la contabilidad de operaciones con la utilización de cuentas de viaje, aunque está mejor definida como la apertura de una cuenta independiente por cada operación:

It was the custom of merchants (...) to open a separate account for each venture or lot of merchandise. Such accounts were charged with all outlays, costs, and expenses and credited with proceeds from sales. The difference remaining after conclusion of the venture represented either a profit or a los and was usually transferred to an account, "Profit and Loss on Merchandise" (Avanzi e disavanzi di mercatantie). Thus profits from the trade and from exchange were kept separate $^{43}$.

Ni la compañía Datini ni la catalana de Marco Serrainerio utilizaron cuentas de viaje. En la organización centralizada de los Datini, el registro de los envíos estaba distribuido en numerosos libros interrelacionados. Cuando la mercancía que se compraba para su envío se embarcaba, los costes, que se cargaban en una cuenta de mercaderías, se cargaban a la delegación a la que se enviaba la mercancía. Otro caso lo encontramos en un negocio conjunto (joint venture) para enviar paño de Pisa a Palermo, del que la cuenta de mercaderías, en la que se habían cargado los costes, se abonó con la cantidad recibida de las ventas en Palermo, ventas que habían efectuado los participantes en el negocio conjunto. La cuenta de mercaderías se cerró con el cálculo del beneficio, del que la mitad se asentó como abono en la cuenta conjunta de propietarios $^{44}$.

Del mismo modo, en el Libro Mayor de la compañía catalana de Serrainerio, en las cuentas de mercaderías se cargaban los costes de adquirir la mercancía y de enviarla; se abonaban las cantidades de su venta con los cargos correspondientes en la cuenta del factor, al que, igualmente, se le cargaban los costes que se le habían generado a un

43 Raymond de Roover, The Rise and Decline of the Medici Bank, 1397-1494, Cambridge, Massachusetts: Harvard University Press, 1963, p. 148.

44 Cfr. Federigo Mellis, Documenti per la storia economica dei secoli XIII-XVI, ed. cit., Documento 127. 
factor intermediario en la travesía. Cuando se producía una pérdida en un envío de fustán, se abonaba en la cuenta del factor, su hermano, y se cargaba en el de pérdidas y ganancias. En ocasiones, al factor se le cargaba con el coste de la mercancía enviada y el abono correspondiente se anotaba en la cuenta de mercaderías. Según se puede apreciar en lo que acabamos de decir, las partidas de las cuentas de los factores eran absolutamente heterogéneas ${ }^{45}$.

La contabilidad veneciana, en fin, también registraba las inversiones en sociedades temporales de ámbito bien definido, como fletar y operar una galera para un viaje concreto. Esos negocios conjuntos se registraban en los libros de los inversores lo mismo que se hacía con la propiedad de la parte correspondiente de la mercancía que se poseía de forma solidaria y mancomunada, según podemos apreciar en las partidas del Libro Diario de Lorenzo Priuli, a comienzos del siglo XVI. En estos negocios conjuntos o joint ventures venecianos los derechos $y$ obligaciones de los socios se sustanciaban en una cosa determinada: mercancía especificada, un barco su fletamento, un préstamo o contrato para impuestos agrícolas:

The investment of the Priuli in the Flemish galleys of 1504 consisted not only in their share in the galley company but also in the use of family funds to buy cargo. They purchased in three ways -individually, jointly with other members of the galley company, and jointly with all the shareholders of the galleys of the fleet. By far the largest outlay was made directly by the family through the purchase for its account of wool, cloth, and oxhides worth about 10.000 ducats. This may be considered the center of the whole venture from the point of view of the Priuli. Collections from the sales of the wool were to be a main income of the family for some time. Their investment in the galley company was very probably a subordinate investment for the purpose of being sure of getting the wool ${ }^{46}$.

Los libros venecianos registran las pérdidas y las ganancias de diversas inversiones sin preocuparse de asignarlas a periodos

45 Tommaso Zerbi, Il mastro a partita doppia di una azienda mercantile del Trecento, Como: Università Commerciale L. Bocconi, Istituto di Ricerche Tecnico Commerciali, 1936, pp. 72-73; 122-123; 256-257.

ed. cit., p. 49.

46 Fredric C. Lane, Venice and History. The Collected Papers of Frederic c. Lane, 
temporales determinados. En otro orden de cosas, no hemos encontrado ni tenemos noticia de que nadie lo haya hecho-, ejemplos de registros de capital y de pérdidas y ganancias de una sociedad total general, aunque sí parece que este tipo de sociedades existió en Venecia. No obstante, no debemos olvidar que Badoer consideró su actividad en Constantinopla como una entidad contable diferenciada del resto de su patrimonio e ingresos. A su vez, por lo que se deduce de las partidas de un libro recopilado por los albaceas de Almorò Pisani, los libros que registran los activos y las operaciones de las compañías filiales de los hermanos Pisani, en 1528 , se llevaron por separado ${ }^{47}$. Los tratados teóricos del siglo XVI nos dan ejemplos de cómo cerrar los libros en un espacio de tiempo relativamente corto, describiendo operaciones poco realistas. $Y$ si tenemos en cuenta la organización de las empresas comerciales venecianas, su contabilidad no suministraba los datos necesarios para la preparación periódica de los balances:

Le forme associative nel commercio dei veneziani non implicano la partecipazione totale dei soci, non ne limitano minimamente l'attività al di fuori della compagnia. In realtà sono società con lo scopo di portare a termine uno o più affari predeterminati, spartendone l'utile, ma senza che questo impedisca ad alcuno dei soci non solo di trattare contemporaneamente affari, anche della stessa specie, per conto suo, ma addirittura di participare ad altre società nello stesso tempo, cosa incocepibile negli ordinamenti toscani. L'independenza negli affari di ogni singolo veneziano giungeva al punto che anche il dependente (che, ovviamente, non aveva mai l'importanza del fattore) poteva esercitare il commercio in propio mentre, contemporaneamente, prestava servizio per il suo datore di lavoro. È ovvio, che, in questa situazione, il rendiconto tra i soci concerne solo l'affare o gli affari compiuti in comune e chiude la vita della società: esso pertanto, anche se può essere considerato un bilancio, è un bilancio di liquidazione, e non di esercizio, perché quest'ultimo presuppone la continuazione dell'organismo aziendale ${ }^{48}$.

Aunque el desarrollo veneciano de la contabilidad de operaciones tenía el inconveniente de no disponer de los datos necesarios

47 Ibid., pp. 39-44.

48 Flavio Pilla, "Il bilancio di esercizio nelle aziende private veneziane", Studi veneziani, XVI (1974), pp. 243-278; 274-275. 
para la preparación periódica de los balances, tenía otras ventajas. El efecto de personificar el viaje, lo mismo que se había hecho con la caja y las mercaderías, fue, por una parte, separar los registros de los cambios en el valor de los activos, enviados o vendidos, del registro de costes de adquisición y envío de la mercancía y, por otra, independizar el registro de liquidación con el factor por lo recaudado por la venta. La cuenta personal del factor presentaba una idea precisa de lo que adeudaba por el cargamento recibido y lo que se le adeudaba por sus envíos, cuyos detalles aparecían como cargos en las correspondientes cuentas de mercaderías.

Cabe preguntarnos: ¿qué ventajas había en agrupar los cargos y abonos en una cuenta de embarque o viaje independiente de los cargos y abonos de la cuenta del factor? Agrupar el registro de costes y beneficios de cargamentos anteriores de determinadas mercancías a destinos concretos, ¿podía ayudar a tomar decisiones sobre futuras inversiones? Esta es una posibilidad que en su día consideró el Profesor Yamey, si bien descartó al considerar que esta separación no se realizó para disponer de una guía de futuras inversiones, sino para llevar un registro detallado de activos y pasivos:

Success depends not on the availability of accounting records in a particular form, but on the ability to perceive possibilities of favourable price movements, the willingness to risk resources on these possibilities, and flexibility in the deployment of resources. Much turns on rapid assimilation and interpretation of available market information (...). The systematic accounting of past business results has a decidedly limited part to play in business decision making ${ }^{49}$.

Cuando se gestó el sistema contable de operaciones, las indicaciones que las pérdidas o ganancias de un viaje concreto podían dar sobre un lugar, de cara a una futura inversión, eran más bien escasas, pues las condiciones comerciales cambiaban con rapidez.

Un objetivo más verosímil de la cuenta de viaje es el de descargar la cuenta personal del factor con el fin de que refleje una visión más clara de sus operaciones y obligaciones. Libre de detalles sobre los gastos de embarque, su cuenta personal presentaba en columnas

49 B.S. Yamey, "Accounting and the Rise of Capitalism: Further Notes on a Theme by Sombart," ed. cit., pp. 129-130. 
opuestas tabuladas el propio informe del factor sobre lo que debía por cargamentos recibidos y lo que se le adeudaba por remesas enviadas o pagos efectuados mediante letras de cambio o transferencia a terceras partes según las instrucciones recibidas. En la cuenta personal del factor también se asentaban los cargos por errores que un consignador hubiera podido detectar en el informe de ventas o compras de su factor, del que existía una anotación completa en la correspondiente cuenta de operaciones o de mercaderías.

\section{VI}

En resumen: es posible que las cuentas de viaje (viaggio) se idearan como una herramienta de gestión para negociar con los factores. También es probable que se utilizaran para orientarse en futuras inversiones; no referentes a lugares y mercancías, sino relacionadas con la elección del factor al que se le fueran a enviar los cargamentos. Su utilidad para ese objetivo dependía de que hubiera un número de factores suficiente en el mercado de destino para elegir. Además, también sirvieron para aclarar las demandas en caso de litigios legales y, sobre todo, en la eventualidad de que una de las partes falleciera. En los siglos XIV y XV la muerte era una contingencia que todo buen sistema gerencial debía tener previsto.

El papel destacado que desempeñaron los factores a comisión y los negocios conjuntos o joint ventures en la organización comercial veneciana hicieron de las cuentas de viaje un instrumento de mucha utilidad. Las compañías que disponían de delegaciones propias en el extranjero, como los Medici de Florencia o los Borromeo de Milán dependían de otros métodos contables:

... the Medici insisted that branch managers send them every year the balance sheet, which was carefully scrutinized upon reaching headquarters. Medieval statements were not systematically arranged as are modern balance sheets; they listed separately debit and credit balances in the order in which the accounts appeared in the ledger, without any further attempt at classification. Some of the Medici balance sheets have two hundred items or more and are booklets of several pages. The audit consisted in going over the balance, item by item, to pick out any dubious or past due accounts. A thorough checking required the presence of the branch manager to answer questions. This is one of 


\begin{abstract}
the reasons why branch managers were called to Florence once every year when residing in Italy and at least once every two years when residing beyond the Alps (...). It was a constant policy of the Medici to make due provision for bad debts before proceeding with the distribution of profits. Reserve accounts were set up for this purpose as is done today. A branch manager was severely reprimanded if it was discovered later that reserves were inadequate because of misleading reports about the solvency of debtors. It was also a standard practice of the Medici to open accounts of accrued salaries and wages ${ }^{50}$.
\end{abstract}

Es indudable que el desarrollo de la partida doble fue, a la vez, causa y efecto de la transición de los mercaderes itinerantes a sedentarios. La expansión del comercio y del número de inversiones en las que un mercader podía estar inmerso a un mismo tiempo creó la necesidad de una integración y un ordenamiento que, en cierto modo, la partida doble exigía, concitaba y propagaba. Y como pasa con el binomio de mejores técnicas en la navegación y expansión del mercado, las relaciones causales son recíprocas. Así, un comercio más amplio demandaba un sistema gerencial mejor; ese buen sistema gerencial fue un factor decisivo en el incremento comercial. $Y$, en efecto, eso es lo que sucedió.

\title{
BIBLIOGRAFÍA
}

BADOER, Giacomo (1956) El libro dei conti di Giacomo Badoer (Constantinopoli, 1436-1440). Testo a cura di Umberto Dorini e Tommaso Bertelè. Roma: Librería dello Stato.

DE ROOVER, Raymond (1953) "The Commercial Revolution of the Thirteenth Century". Frederic C. LANE and Jelle C. RIMERSMA (eds.) Enterprise and Secular Change. Readings in Economic History. London: George Allen and Unwin Ltd., pp. 80-85.

- (1955) "New Perspectives on the History of Accounting," The Accounting Review, XXX, pp. 405-420.

cit., p. 100.

50 Raymond de Roover, The Rise and Fall of the Medici Bank, 1397-1494, ed. 
- (1963) "The Organization of Trade." M.M. POSTAN, E.E. RICH and Edward MILLER (eds.) The Cambridge Economic History of Europe. Economic Organization and Policies in the Middle Ages. Cambridge University Press, vol. III.

- (1963) The Rise and Decline of the Medici Bank, 1397-1494. Cambridge, Mass.: Harvard University Press.

- (1974) "The Development of Accounting Prior to Luca Pacioli According to the Account Books of Medieval Merchants". Julius KIRSCHNER (ed.) Business, Banking and Economic Thought in Late Medieval and Early Modern Europe. University Press of Chicago, pp. 161-164.

EDLER DE ROOVER, Florence (1966) "Andea Bianchi, Florentine Silk Manufacturer and Merchant in the Fifteenth Century." William $M$. BowSLY (ed.) Studies in Medieval and Renaissance History. Lincoln, Nebraska: University of Nebraska Press, vol III, pp. 223-285.

GRAS, N.S.B. (1953) "Capitalism -Concepts and History". Frederic C. LANE and Jelle C. Riemersma (eds.) Enterprise and Secular Change. Readings in Economic History. London: George Allen and Unwin Ltd.

KEDAR, Benjamin Z. (1976) Merchants in Crisis: Genoese and Venetian Men of Affairs and the Fourteenth-Century Depression. New Haven and London: Yale University Press.

KeLlebenz, Hermann (1974) "Technology in the Age of Scientific Revolution 1500-1700." Carlo M. CIPOLLA (ed.) The Fontana Economic History of Europe. The Sixteenth and Seventeenth Centuries. Glasgow: William Collins Sons \& Ltd., vol. 2.

KImURA, Wasaburo (1975) "Double-Entry Bookkeeping and Business Bookkeeping." Osamu KoJIMA (ed.) Historical Studies of Double Entry. Kyoto: Daigakudo Shoten Limited, pp. 259-260.

KoJIMA, Osamu (1995) Accounting History. Nakatomigaoka, Japan: Kohko Kojima.

LANE, Frederic C. (1944) Andrea Barbarigo, Merchant of Venice, 14181449. Baltimore: The Johns Hopkins Press.

- (1966) Venice and History: The Collected Papers of Frederic C. Lane. Baltimore: The Johns Hopkins Press.

- (1973) Venice, A Maritime Republic. Baltimore: John Hopkins University Press.

- (1977) "Double Entry Bookkeeping and Resident Merchants," Journal of European Economic History, 6, pp. 177-191. 
- and Jelle C. RIEMERSMA (eds.) (1953) Enterprise and Secular Change. Readings in Economic History. London: George Allen and Unwin Ltd.

LANERO FERnÁNDEZ, J. y E. ORTEGA MONTES (2007) "De aprendiz a mercader: El factor en el comercio internacional inglés del siglo XVI", Pecvnia, pp. 145-180.

LOPEZ, Robert S. (1976) The Commercial Revolution of the Middle Ages, 950-1350. Cambridge University Press.

- (1987) "The Trade of Medieval Europe: The South." The Cambridge Economic History of Europe, vol. II: Trade and Industry in the Middle Ages, Ed. by M.M. Postan and Edward Miller. Cambridge University Press.

LUZZATTO, Gino (1961) Storia economica di Venezia dall'XI al XVI secolo. Venezia: Centro Internazionale delle Arti e del Costume.

MELLIS, Federigo (1962) Aspetti della vita economica medievale (Studi nell'archivio Datini di Prato). Firenze: Leo S. Olschki.

- (1972) Documenti per la storia economica dei secoli XIII-XVI, con una nota di Paleografia Commerciali a cura di Elena Cecchi. Istituto Internazionale di Storia Economica "F. Datini", Prato. Publicazioni-Serie I, Documenti. Firenzi: Leo S. Olschki.

Paton, W.A. (1922) Accounting Theory. New York: The Ronald Press Company.

PegolotTI, Francesco Balducci (1936) La pratica della mercatura. Ed. by Allan Evans. Cambridge, Mass.: The Medieval Academy of America.

PILLA, Flavio (1974) "Il bilancio di esercizio nelle aziende private veneziane", Studi veneziani, XVI, pp. 243-278.

ReNOUARD, Yves (1949) Les hommes d'affaires italiens du Moyen Âge. Paris: Librairie Armand Colin.

SAPORI, Armando (1946) Studi di Storia Economica Medievale. Firenze: G.C. Sansoni.

SOMBART, Werner (1928) Der moderne Kapitalismus. Historischsystematische Darstellung des gesamteuropäischen Wirtschaftslebens von seinem Anfängen bis zur Gegenwart. München und Leipzig: Verlag von Duncker \& Humblot.

Tuccl, Ugo (1968) "Tariffe veneziane e libri toscani di mercatura", Studi veneziani, $\mathrm{X}$, pp. 65-108.

YAMEY, B.S. (1949) "Scientific Bookkeeping and the Rise of Capitlism," Economic History Review, 1, pp. 99-113. 
- (1964) "Accounting and the Rise of Capitalism: Further Notes on a Theme by Sombart," Journal of Accounting Reserch, 2 (Spring), pp. 117-136.

- (1975) "Notes on Double-Entry Bookkeeping and Economic Progress," Journal of European Economic History, IV, 3 (Winter), pp. 717-723.

- (2005) "The Historical Significance of Double-Entry Bookkeeping: Some non-Sombartian Claims", Accounting, Business \& Financial History, 15 (1), pp. 77-88.

ZERBI, Tommaso (1936) Il mastro a partita doppia di una azienda mercantile del Trecento. Como: Università Commerciale L. Bocconi, Istituto di Ricerche Tecnico Commerciali.

- (1952) Le origini della partita doppia: Gestioni aziendali e situazioni di mercato nei secoli XIV e XV. Milan: Marzorati. 